\title{
ANALYSIS OF RESONANT TUNNELING FOR CdTe-CdMgTe STRUCTURES
}

\author{
E. KACZMAREK \\ Institute of Physics, Polish Academy of Sciences \\ Al. Lotników 32/46, 02-668 Warszawa, Poland
}

\begin{abstract}
In this paper we present model calculations of the current-voltage characteristics for the $\mathrm{CdTe} / \mathrm{CdMgTe}$ double barrier structures based on the assumption that the electron effective masses in the barrier and well regions of double barrier structure are different. The main features of the measured $I-V$ characteristics, i.e., the small current peak at low bias and much larger peak at high voltage, are reproduced quite well by the calculated curve. The results of magnetotunneling experiments can be also understood in the frame of the proposed model.
\end{abstract}

PACS numbers: $73.40 . G k$

Since its first observation in GaAs/GaAlAs double barrier structure (DBS) [1], the resonant tunneling has been almost exclusively studied in III-V materials. Recently, the resonant tunneling has been also observed in $\mathrm{CdTe} / \mathrm{CdMgTe}$ structures $[2,3]$. The general features of the current-voltage characteristics for these structures are similar to those for GaAs/GaAlAs structures, but the detailed analysis of the characteristics cannot be done without a better knowledge of the structure parameters. The authors performed several experiments in order to determine these parameters. First, the barrier height $V_{b}$ was evaluated from the slope of the peak current density versus barrier width under assumption that the effective mass $m_{\mathrm{b}}$ of the CdMgTe is equal to the effective mass of CdTe. Secondly, the energetic distance between the Fermi level in the emitter, $E_{\mathrm{F}}$, and the first subband in the quantum well was determined in two ways: from the Arrhenius plot for resonant tunneling structure [4] and from magnetotunneling measurements performed at constant magnetic field parallel to the current. Unfortunately, the interpretation of these experiments gives inconsistent results. Namely, the emitter Fermi energy $E_{\mathrm{F}}$ calculated from the Arrhenius plot is equal to $50 \mathrm{meV}$ whereas the value of $E_{\mathrm{F}}$ deduced from the magnetotunneling experiments is an order of magnitude smaller. Here we propose another interpretation of the experiments done for the $\mathrm{CdTe} / \mathrm{CdMgTe}$ resonant structures, which is based on the assumption of different masses in the DBS barrier and well regions.

To show how the "barrier mass", i.e., how the difference of barrier and well masses, influences the resonant tunneling we use the most elementary example of 
two rectangular barriers with the same heights. The global transmission coefficient, $T_{\mathrm{G}}$, for such structure has been calculated in our earlier paper [5]. The barrier mass enters the expression for $T_{\mathrm{G}}$ in two ways: through the condition of the continuity of the quantum current density and through the imaginary wave vector in the barrier region. The approximate condition for resonance can be expressed as

$$
w \sqrt{\frac{2 m_{\mathrm{w}} E}{\hbar^{2}}}=2 \arctan \sqrt{\frac{m_{\mathrm{w}}}{m_{\mathrm{b}}}\left(\frac{V_{\mathrm{b}}-E}{E}\right)}+n \pi .
$$

Here $m_{\mathrm{w}}$ and $m_{\mathrm{b}}$ stand for the electron effective mass in the well and barrier regions, respectively, $w$ is the well width, $V_{\mathrm{b}}$ is the barrier height and $E$ denotes the energy of the tunneling electron.

Generally, the barrier mass causes the lowering of the resonance energies. The dependence of resonance energy on the barrier-to-well mass ratio at fixed value of $m_{\mathrm{b}} V_{\mathrm{b}}$ is shown in Fig. 1 for two CdTe-CdMgTe structures. The structure parameters are: for the sample CT1141 the barrier width $b$ is equal to $5 \mathrm{~nm}$ and the well width $w=4 \mathrm{~nm}$, and for the sample CT1198 $b=8 \mathrm{~nm}$ and $w=5 \mathrm{~nm}$ [6]. The energetic distance $\Phi$ between the Fermi level in the emitter, $E_{\mathbf{F}}$, and the first subband in the quantum well, as determined from the Arrhenius plot, is also shown in the figure. For $m_{\mathrm{b}}=m_{\mathrm{w}}$ this gives for the emitter Fermi energy the value $E_{\mathrm{F}}^{\prime} \approx 50 \mathrm{meV}$. This value corresponds to the carrier concentration equal to $2 \times 10^{18} \mathrm{~cm}^{-3}$. The nominal carrier concentration for these structures at the regions adjacent to the DBS structure is $10^{17} \mathrm{~cm}^{-3}\left(E_{\mathrm{F}} \approx 10 \mathrm{meV}\right)$. It is well known that the carrier concentration may be slightly larger than the nominal one due to the dopant diffusion in the molecular-beam epitaxy growth direction (for GaAs the real concentration of carriers in the $n$-type emitter layer is estimated to be two or three times larger than the nominal one), but it is hard to believe the diffusion to be large enough to explain one order of magnitude difference in the nominal and observed concentrations.

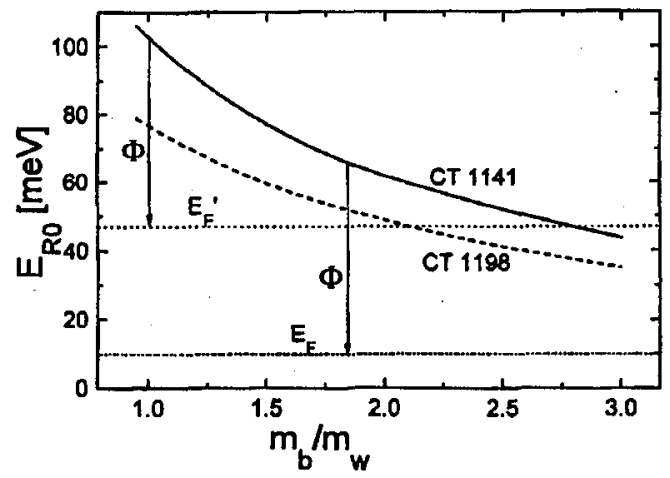

Fig. 1. Resonance energies $E_{\mathrm{R} 0}$ as a function of the barrier-to-well mass ratio for $\mathrm{CdTe} / \mathrm{CdMgTe}$ double barrier structures. The parameters of the structures are given in the text. 


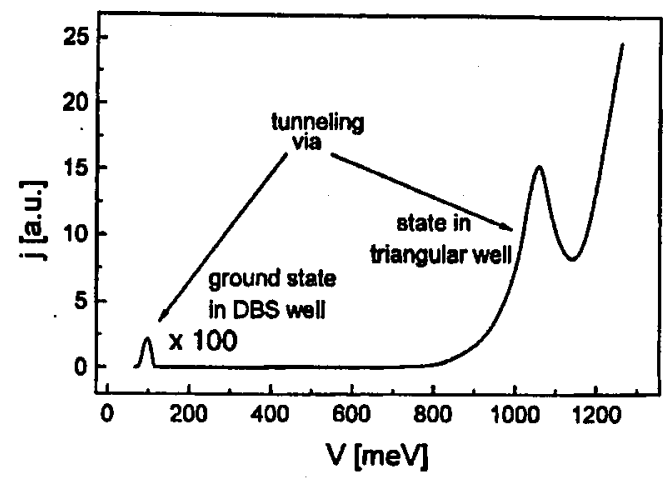

Fig. 2. The $j(V)$ characteristic calculated for the CT1198 sample. The parameters of the structures are given in the text.

Thus, in the following we assume that the electron effective mass for CdMgTe is larger than that for CdTe. We take $m_{\mathrm{b}}=0.17 m_{0}$ and $m_{\mathrm{w}}=0.095 m_{0}$. The barrier height $V_{\mathrm{b}}$ calculated from the experimentally determined slope of the peak current density versus barrier width is then equal to $0.23 \mathrm{eV}$. The current-voltage characteristic, $j(V)$, calculated for the double barrier structure with this set of parameters and $E_{\mathrm{F}}=15 \mathrm{meV}$ is plotted in Fig. 2. The main features of the measured characteristic, i.e., the small current peak at low bias and much larger peak at high voltage, are reproduced quite well by the calculated curve. However, only the first peak corresponds to the resonant tunneling through the state in the DBS well whereas the second one corresponds to the Fowler-Nordheim tunneling through the resonant state in the triangular well formed over the second barrier of the DBS structure.

Now let us discuss briefly the relation between the results of the magnetotunneling experiments and the result of our model calculations. No significant change in $j(V)$ characteristics was observed for different values of the magnetic field parallel to the current direction. This indicates large broadening of the Landau levels. At constant voltage the oscillations were measured for the two bias voltages corresponding to the beginning of resonant tunneling through the ground state in the well and to the current maximum. The results allow us to estimate the Fermi energy for the structure. Moreover, they indicate that the resonant tunneling occurs in a narrow energy range, in agreement with our model calculations.

Finally we comment concisely the problem of spin-splitting in the resonant tunneling. It is well known [7] that the exchange term of the Coulomb interaction can lead to a drastic enhancement of the spin splitting in 2DEG systems. In strong magnetic fields the density of states becomes discrete and the difference in numbers of electrons-with $\uparrow$ and $\downarrow$ spins, to which the spin-splitting is proportional, depends strongly on the position of the Fermi level. The Fermi level situated half-way between $(N, \uparrow)$ and $(N, \downarrow)$ levels leads to the biggest difference in the occupation of these levels and to the maximum value of the effective $g^{*}$ factor which is about two orders of magnitude larger than the bare $g$ factor. When the Fermi level lies 
midway between the $(N, \downarrow)$ and $(N+1, \uparrow)$ levels, the difference becomes minimal and the $g^{*}$ has a minimum value close to $g$. Thus, the enhanced spin-splitting may affect maxima of $j(B)$ curve, whereas the minima should remain unchanged.

The enhanced spin-splitting was observed for GaAs/GaAlAs heterostructures in transverse magnetoresistance [8] and it was not observed in tunneling, neither for tunneling from accumulation layer [9] nor for resonant tunneling [10]. Probably this is due to the large broadening of Landau levels in these structures. This problem requires, however, a more detailed analysis.

This work was partially supported by the Committee for Scientific Research, grant No. 2 P03B 03508.

\section{References}

[1] L.L. Chang, L. Esaki, R. Tsu, Appl. Phys. Lett. 24, 593 (1974).

[2] G. Reuscher, M. Keim, F. Fischer, A. Waag, G. Landwehr, Acla Phys. Pol. A 88, 885 (1995).

[3] G. Reuscher, M. Keim, F. Fischer, A. Waag, G. Landwehr, Phys. Rev. B 53, 16414 (1996).

[4] P. Gueret, C. Rossel, E. Marclay, H. Meier, J. Appl. Phys. 66, 278 (1989).

[5] E. Kaczmarek, W. Szkielko, Acta Phys. Pol. A 80, 441 (1991).

[6] M. Keim, private communication.

[7] A. Manolescu, R.R. Gerhardts, Phys. Rev. B 51, 1703 (1995) and references therein.

[8] Th. Englert, D.C. Tsui, A. Gossard, Ch. Uihlein, Surf. Sci. 113, 295 (1982).

[9] E. Bockenhoff, K. von Klitzing, Phys. Rev. B 38, 10120 (1988).

[10] E. Bockenhoff, K. von Klitzing, Surf. Sci. 229, 182 (1990). 